\title{
A Picture is Worth a Thousand Words but Quantitation is Worth a Thousand Micrographs.
}

\author{
W. Gray (Jay) Jerome ${ }^{1}$ \\ ${ }^{1}$ Department of Pathology, Microbiology and Immunology, Vanderbilt University School of Medicine, \\ Nashville, TN, USA
}

A picture is certainly worth a thousand words and a great micrograph is probably worth even more. However, microscopists often look at thousands of fields in order to draw conclusions about what a single micrograph means. This is because we need to determine and assess the natural variability that occurs in nature and also how our preparation techniques affect variability either of our sample or our observation of the sample. For instance, thin sectioning removes information about how observed structures relate to objects above and below the plane of section and we only see interactions if they are within the plane of section. Thus, the act of thin sectioning adds additional variability to our observations. At the end of all of our observations and assessment of what it means, for presentation or publication we have to choose one "representative" micrograph that summarizes all of our hard work. Moreover, in most situations our micrographs are much more meaningful if we can ascribe functional significance to our structural findings. Quantification of structural information can help with all of these conundrums. Quantification allows the microscopist to summarize data from a large number of micrographs and confirm that our "representative" micrograph is truly representative. Quantification also allows us to assess variability and determine if our conclusions could be in error because we have not observed a sufficient number of fields to rule out variability. Quantification can also point out subtle differences that our eye does not detect. Quantification of structural information can also allow us to directly correlate structure with functional data that is often quantitative in nature. Finally, quantification provides a translation language for comparing information derived from microscopes with vastly different size scales (e.g. photon and electron microscopes).

Although quantitation of structure would seem fairly straightforward, it usually is not and, unfortunately, a large number of papers with incorrect quantitative methods are published each year. At best, these poor studies do not exploit the full potential of quantification; at worst they produce spurious conclusions. One of the issues is that our micrographs depict 3D structure in 2D, thus without laborious $3 \mathrm{D}$ reconstruction, we have lost depth which is critical for correct quantification. In this presentation, I will review some of the pitfalls of quantification and review some basic procedures for simply but effectively drawing quantitative conclusions from 2D micrographs. The principals are relevant for both biological and material science studies but the illustrations will come from studies conducted in my laboratory over the years on atherosclerosis, blood clotting and intracellular cholesterol metabolism.

One such study involved examining why blood clots formed on the surface of endothelial cells were harder to dissolve than those formed in the absence of endothelial cells. A principal component of blood clots are fibrin fibers formed when small fibrinogen molecules are stimulated to coalesce into long fibers. Figure 1A shows an SEM image of fibrin fibers formed in a tube that was coated with endothelial cells (EC). Blood was pumped through the tube and clot formation stimulated by introducing thrombin into the tube. In Figure 1A you can see the endothelial cells below the fibers. The fibrinogen in the blood was labeled with fluorescent Oregon green. Figures 1B and C show confocal images of the blood clot at the surface of the endothelial cells. Figure $1 \mathrm{C}$ differs from Figure $1 \mathrm{~B}$ in that in $1 \mathrm{C}$ an RGD 
peptide was included that blocks binding of fibrin to integrins on the endothelial cell surface. Figure 1D shows the quantification of the mean fluorescence intensity per field and the variation in pixel intensities from 100 fields. The means for clots formed in the presence or absence of RGD are very similar. However, the variation is much greater for endothelial cells without RGD. What we were able to show from this is that the same amount of fibrin was seen in each field but that binding to integrins on the endothelial surface organized the fibrin fibers into thicker bundles (thus there were a lot more pixels without fluorescence which produced the greater variability in individual pixel intensity). In later studies we were able to show that because of the organization into thicker fibers, the fibrin fibers formed on the endothelial surface were more resistance to "clot busting" drugs because the drugs could not penetrate between the fibers and dissolve the clot. Other examples of how to efficiently but correctly determine size of objects, shapes of objects and number of objects from microscope images will be provided.

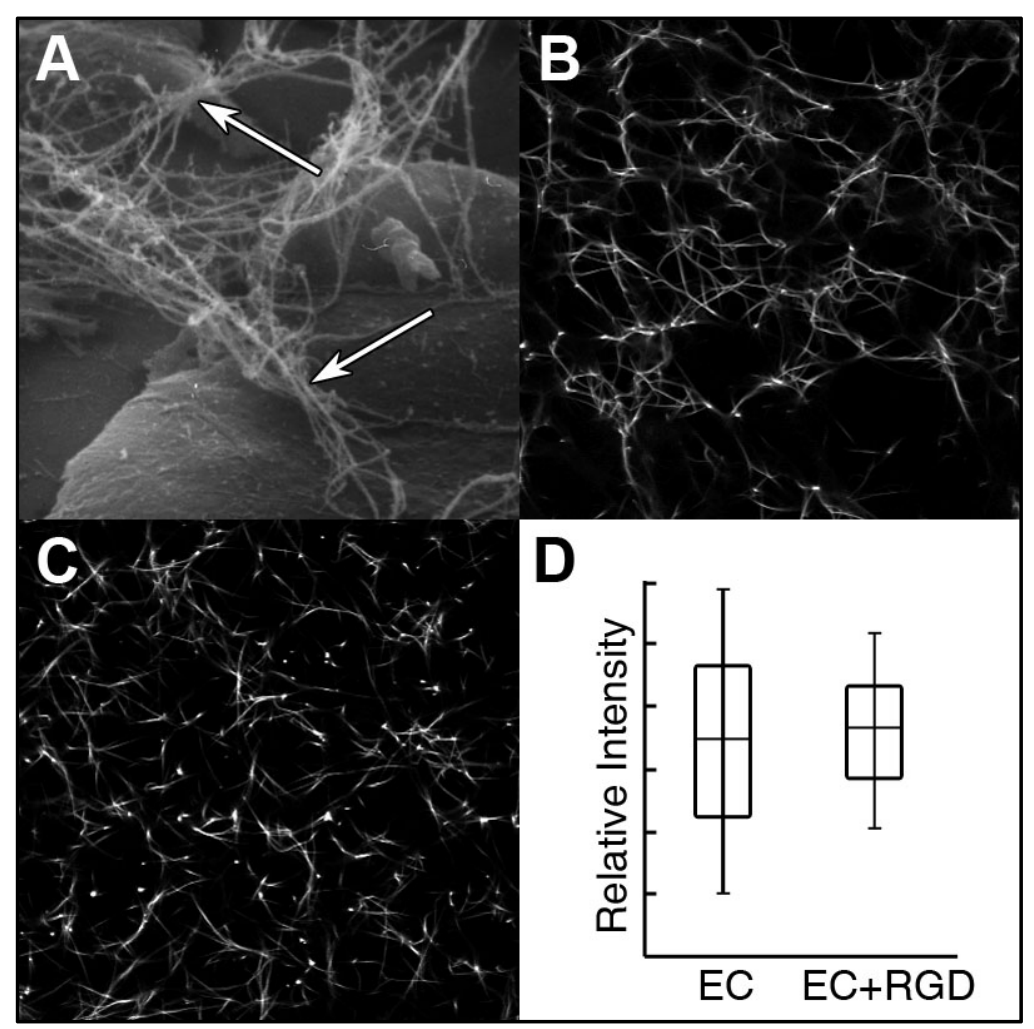

Figure 1: Fibrin binding to integrins on endothelial cells organize the clot fibers into tight bundles. A) SEM of fibrin fibers (arrows) formed on surface of endothelial cells. B) Flourcent fibrin clot formed on surface of endothelial cells. C) Flourescent fibrin clot formed on surface of endothelial cells in the presence of RGD peptide. D) Mean and variance of individual pixel intensities per field. 